\title{
Transient Spine Expansion and Learning-Induced Plasticity in Layer 1 Primary Motor Cortex
}

\author{
Kimberly J. Harms, ${ }^{\star}$ Mengia S. Rioult-Pedotti, ${ }^{\star}$ D. Rosy Carter, and Anna Dunaevsky \\ Department of Neuroscience, Brown University, Providence, Rhode Island 02912
}

\begin{abstract}
Experience-dependent regulation of synaptic strength in the horizontal connections in layer 1 of the primary motor cortex is likely to play an important role in motor learning. Dendritic spines, the primary sites of excitatory synapses in the brain, are known to change shape in response to various experimental stimuli. We used a rat motor learning model to examine connection strength via field recordings in slices and confocal imaging of labeled spines to explore changes induced solely by learning a simple motor task. We report that motor learning increases response size, while transiently occluding long-term potentiation (LTP) and increasing spine width in layer 1. This demonstrates learning-induced changes in behavior, synaptic responses, and structure in the same animal, suggesting that an LTP-like process in the motor cortex mediates the initial learning of a skilled task.
\end{abstract}

Key words: dendritic spines; motor cortex; layer 1; learning; LTP; field potentials

\section{Introduction}

Experience-dependent regulation of synaptic strength may be the physiological basis of learning and memory. Most excitatory synapses in the brain form on dendritic spines, small protrusions from dendrite shafts, which are important loci for encoding synaptic plasticity. Dendritic spine number and morphology can change with synaptic activity, sensory experience, and induction of long-term potentiation (LTP) or long-term depression (LTD) (Bourne and Harris, 2007). Synaptic strength correlates with spine size, thus changes in spines may be linked to changes in synaptic function (for review, see Harms and Dunaevsky, 2007). However, studies investigating cortical plasticity and spine dynamics largely focus on sensory deprivation paradigms during development, whereas few studies examine modification of spines with learning in the adult and how this relates to synaptic strength.

Layer 1 of the primary motor cortex contains few neurons but has a prominent horizontal system of axons originating from multiple cortical and subcortical regions. These inputs form a high density of synapses on the apical dendrites of deeper pyramidal cells (Douglas and Martin, 2004). Converging inputs into layer 1 may be important for integration of information from thalamic and higher-order cortical areas (Cauller, 1995), and could be critical in cortical reorganization with learning (Sanes and Donoghue, 2000).

Acquisition of a new motor skill is associated with enhancement of synaptic strength in the primary motor cortex (Rioult-

\footnotetext{
Received Feb. 8, 2008; revised April 21, 2008; accepted April 22, 2008.

This work was supported by National Institute of Mental Health Grants MH019118-14 and 1R21MH073852-01. We thank Karen Kudelko, Heather Johnston, and David Coughlin for technical assistance.

${ }^{*}$ K.J.H. and M.S.R.-P. contributed equally to this work.

Correspondence should be addressed to Anna Dunaevsky, Department of Neuroscience, Brown University, Box G-L459, 185 Meeting Street, Providence, RI 02912. E-mail: Anna_Dunaevsky@brown.edu. D01:10.1523/JNEUROSCI.0584-08.2008

Copyright $\odot 2008$ Society for Neuroscience $\quad$ 0270-6474/08/285686-05\$15.00/0
}

Pedotti et al., 1998; Hodgson et al., 2005). Five days of training on a reach and grasp task (see Fig. 1) enhances layer 2/3 horizontal connections contralateral to the trained forelimb (Rioult-Pedotti et al., 1998, 2000). After training, the ability to induce LTP is partially occluded, whereas LTD is increased (Rioult-Pedotti et al., 2000), suggesting that an LTP-like mechanism mediates learning-induced synaptic strengthening. In contrast, weeks after training is discontinued, both LTP and LTD return to pretraining levels, whereas synaptic connections remain strengthened in the trained hemisphere (Rioult-Pedotti et al., 2007).

To study how spines change with learning, we combined this model, in which learning of a new motor skill induces synaptic strengthening in the motor cortex, with analysis of dendritic spines. The combination of motor skill learning, persistent changes in synaptic strength as shown by slice recordings, and imaging of dendritic spines provides an opportunity to link behavioral changes with synaptic efficacy and structure at two points after training. Here, we report that motor learning stably increases response size while transiently occluding LTP and increasing spine width in layer 1, suggesting that an LTP-like process in motor cortex transiently increases synaptic strength and size to mediate the initial learning, but not long-term retention of a skilled task.

\section{Materials and Methods}

Motor skill training. Animals were cared for in accordance with National Institutes of Health guidelines for laboratory animal welfare. All experiments were approved by the Brown University Institutional Animal Care and Use Committee. Adult female Sprague Dawley rats (8-10 weeks old) housed in groups of three to six were food restricted before training, maintained at $85 \%$ of their ad libitum feeding weight (weight was recorded on a daily basis). Female rats were used in this study because all previous studies using this model characterized the electrophysiological changes in female rats (Rioult-Pedotti et al., 1998, 2000, 2007). In these previous studies, female rats were found to be easier to handle and more adept at learning this particular task in a more consistent manner. Train- 
a

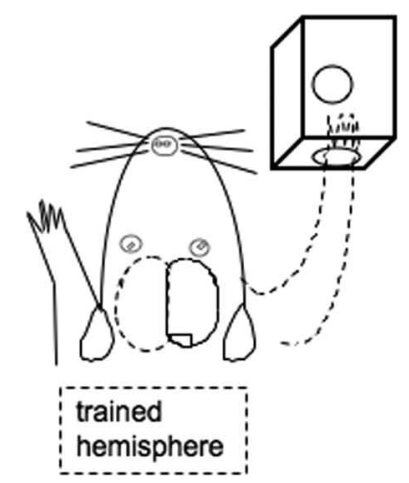

Figure 1. Motor skill training and slice recording. $\boldsymbol{a}$, Rats are trained to reach into a food box with one forelimb and grasp small food pellets. The trained hemisphere is contralateral to the trained paw (dashed). $\boldsymbol{b}$, Extracellular FPs of horizontal connections in $\mathrm{M} 1$ are recorded simultaneously in both hemispheres of coronal slices.

ing was done in a cage $\left(22.8 \mathrm{~cm}^{3}\right)$ containing a Plexiglas food box $(3.2 \times$ $4.5 \times 5 \mathrm{~cm})$ with a $1.3 \mathrm{~mm}$ diameter aperture, through which small food pellets (45 mg; Noyes Precision Food Pellets) could be retrieved. Rats learned to reach into the food box with one forelimb to grasp single food pellets (Fig. 1a). Rats trained for $\sim 1$ h per day for $5-6 \mathrm{~d}$. Rats that switched forelimbs or use their snouts during training were excluded from the analysis. Motor skill performance was quantified by the success rate (ratio of pellets retrieved and consumed to total number of retrieved pellets). Rats were used either 1-3 d after the last day of training, or returned to normal housing for an additional 25-30 d without further training before used for electrophysiological recordings and spine morphology analysis.

Estrous cycle monitoring. To control for fluctuations in learning and spines over the course of the rats' $4-5 \mathrm{~d}$ estrous cycle, vaginal lavage and microscopic determination of vaginal cytology were used to confirm estrous cycle stage (Evans and Long, 1922). Stages of the estrous cycle were monitored daily 5-7 d before initiation of training and before slice preparation for electrophysiology and spine imaging. Training was never begun during proestrous, and the length of training encompasses the entire 4-5 d estrous cycle. Rats were not used for slice preparation during proestrous, a stage shown to increase estrogen levels and spine density. However, we found no difference in spine parameters in monitored and unmonitored rats (supplemental Table 1, available at www.jneurosci.org as supplemental material).

Live slice preparation. Deeply anesthetized rats (Nembutal, $50 \mathrm{mg} / \mathrm{kg}$ ) were decapitated and their brains quickly removed and immersed in cold $\left(5-7^{\circ} \mathrm{C}\right)$, oxygenated $\left(95 \% \mathrm{O}_{2} / 5 \% \mathrm{CO}_{2}\right)$ artificial CSF (ACSF) containing (in $\mathrm{mm}$ ) $126 \mathrm{NaCl}, 3 \mathrm{KCl}, 1.25 \mathrm{NaH}_{2} \mathrm{PO}_{4}, 1 \mathrm{MgSO}_{4}, 2 \mathrm{CaCl}_{2}, 26$ $\mathrm{NaHCO}_{3}$, and 10 dextrose. Coronal slices containing the forelimb motor regions (1.5-3.5 $\mathrm{mm}$ anterior to bregma, 2-4 $\mathrm{mm}$ from the midline) from both hemispheres were cut to $500 \mu \mathrm{m}$ using a vibratome, then transferred to a temperature-controlled $\left(34 \pm 0.5^{\circ} \mathrm{C}\right)$ chamber and superfused with oxygenated ACSF at a rate of $1-2 \mathrm{ml} / \mathrm{min}$.

Stimulation and field potential recordings. Concentric bipolar stimulation electrodes were positioned symmetrically in layer 1 of each hemisphere $2 \mathrm{~mm}$ lateral to the midline, and recording electrodes placed 500 $\mu \mathrm{m}$ laterally (Fig. 1b). Extracellular field potentials (FPs) were evoked by $0.2 \mathrm{~ms}$ pulses at $0.03 \mathrm{~Hz}$ and recorded simultaneously in both hemispheres. Stimulation intensities were adjusted until a response of $0.2 \mathrm{mV}$ was recorded, which was defined as the threshold intensity, and multiples of this intensity were used for determination of input-output relationships (Rioult-Pedotti et al., 1998). Peak amplitudes of the negative-going FP were used as a measure of synaptic strength. Input-output relationships for each hemisphere were generated. All recordings were performed blind to the training condition and hemisphere.

Electrical induction of LTP. The stimulus intensity eliciting $50 \%$ of the maximum amplitude was used for all measurements before and after conditioning. Baseline amplitude was recorded using single stimuli ap- plied to both hemispheres every $30 \mathrm{~s}$. After a 30 min stable baseline period, LTP was simultaneously induced in both hemispheres. LTP was induced by theta burst stimulation (TBS), consisting of 10 trains of $5 \mathrm{~Hz}$ stimuli, each composed of $4(200 \mu \mathrm{s})$ pulses at $100 \mathrm{~Hz}$, repeated five times every $10 \mathrm{~s}$ (supplemental Fig. 2, available at www.jneurosci.org as supplemental material). During TBS, the stimulation intensity was doubled. TBS was applied immediately after transient, local application of the $\mathrm{GABA}_{\mathrm{A}}$ receptor antagonist bicuculline methiodide ( $3.5 \mathrm{mM}$ ) at the field potential recording site until response amplitude was $150-200 \%$ of baseline.

DiI and Nissl labeling. Cortical slices in which LTP was not induced were fixed with $4 \%$ paraformaldehyde for $2 \mathrm{~h}$ and rinsed in $0.1 \mathrm{M} \mathrm{PB}, \mathrm{pH}$ 7.4 , then embedded in $2 \%$ agarose and resectioned on a vibratome to a thickness of $150-200$ $\mu \mathrm{m}$. Sections were labeled using the DiOlistic technique (Gan et al., 2000). DiI (Invitrogen)coated $1.6 \mu \mathrm{m}$ gold particles were shot into slices using a gene gun (Bio-Rad) and incubated $2 \mathrm{~d}$ at $4^{\circ} \mathrm{C}$. Sections were then Nissl stained for $20 \mathrm{~min}$ at room temperature (NeuroTrace 500/525; Invitrogen) and mounted in $65 \%$ glycerol in PB.

Confocal imaging of neurons in fixed slices. All images were acquired blind to the training condition and hemisphere. Layers were identified by Nissl staining and distance from the surface. Labeled apical tuft dendrites of layer I in the motor region (1.5-3.5 mm anterior to bregma, 2-4 mm from the midline) were imaged with a Leica TCS SP2 AOBS microscope with a $63 \times 1.4$ numerical aperture objective at a resolution of $116 \times 116$ $\times 285 \mathrm{~nm}^{3}$.

Image analysis. All analysis was performed blind with regards to the experimental condition and hemisphere. Dendritic branches of mean length of $40.7 \pm 14 \mu \mathrm{m}$ (mean $\pm \mathrm{SD}$ ) were analyzed. Spine density was manually counted while scrolling through the $z$-stack. Maximum intensity projections were made from each $z$-stack to measure spine length and width using MetaMorph (Molecular Devices). Length was measured from shaft to tip using the bent-line tool and width was measured at the widest point of the spine approximately perpendicular to the length using the straight-line tool. Spines parallel to the $z$-axis were excluded from this analysis. All measurements for individual spines were tracked for assessment of covariation.

Statistical analysis. Raw spine measurements (density, width, and length) were analyzed using the SPSS statistics software package. Normality was tested using the Shapiro-Wilkes test, and Kruskal-Wallis (a nonparametric one-way ANOVA) was used to test for the main effect. When an effect was found we used a post hoc Mann-Whitney test (for non-normal data) or $t$ test (for normal data) to look for specific changes between the trained and untrained forelimb motor cortex. Box plots are used to show the distribution. Line in box represents the medians. The lower and upper edges represent the 25 th and 75 th percentiles. The whiskers represent the values at $1.5 \times$ interquartile distance (between 25 th and 75 th percentiles). Note that the whiskers do not represent SD or SEM.

\section{Results}

Consistent with findings in layer $2 / 3$, evoked FP responses in layer 1 of the forelimb region were larger in the trained compared with the untrained hemisphere after $5 \mathrm{~d}$ of motor learning (i.e., $1.1 \pm 0.07 \mathrm{mV}$ trained vs $0.72 \pm 0.05 \mathrm{mV}$ untrained for threshold factor $4 ; n=15 ;{ }^{\star} p=0.03$ ) (Fig. $2 b$, supplemental Fig 1, available at www.jneurosci.org as supplemental material), suggesting a strengthening of connections between primary motor cortex (M1) neurons. After establishing baseline responses, LTP was induced repeatedly until saturated in a subset of these slices (supplemental Fig 2, available at www.jneurosci.org as supplemental material). LTP in layer 1 was reduced in the trained hemisphere 


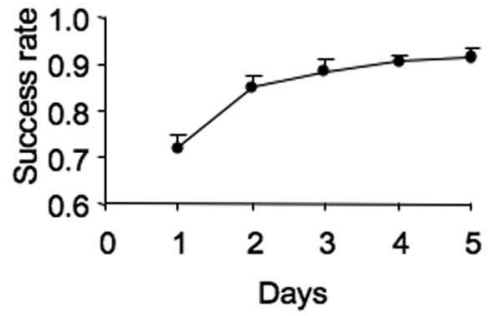

b

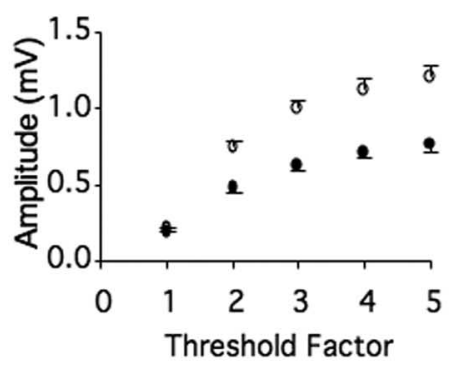

e

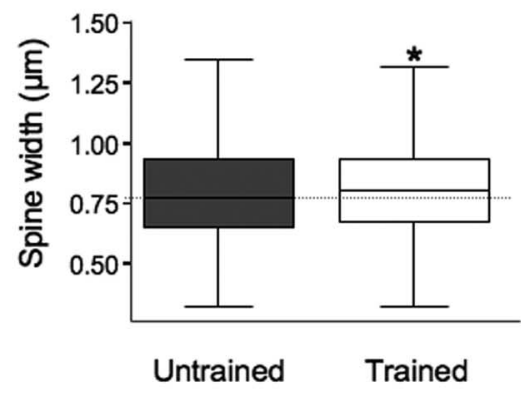

f
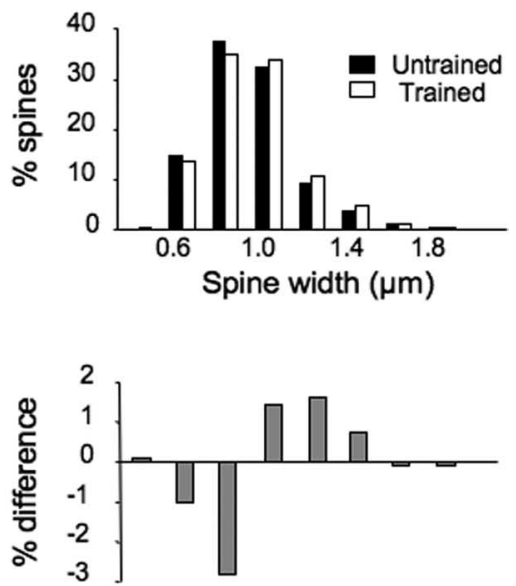

Figure 2. Five days of training increases synaptic responses and spine size and partially occludes LTP. $\boldsymbol{a}$, For $5 \mathrm{~d}$, adult rats were trained to reach with a single forepaw through a small aperture in a box and grasp food pellets. Performance improved over the first $3-4 \mathrm{~d}$ of training, reaching a plateau by day $5 . \boldsymbol{b}$, Extracellular FPs were recorded simultaneously in the forelimb area of both hemispheres of coronal brain slices. FP amplitudes were larger in the trained hemisphere across stimulation intensities as revealed by input- output relationships. $c$, LTP was induced repeatedly (multiple arrows) until responses were saturated in both hemispheres. LTP was reduced in layer 1 of the trained $(\bigcirc)$ compared with the untrained hemisphere $\left(\boldsymbol{O} ; t\right.$ test, $\left.{ }^{*} p=0.016 ; n=5\right)$. Inset, Individual traces before and after LTP saturation. $\boldsymbol{d}$, After recording, slices were fixed, labeled with Dil, and spines in layer 1 imaged with confocal microscopy. $n=6$ rats. Scale bar, $10 \mu \mathrm{m}$. $\boldsymbol{e}$, Analysis of morphology showed spines had a greater spine head width in the trained hemisphere (Kruskal-Wallis, ${ }^{*} p=0.02$ ). Note that the whiskers do not represent SD or SEM. $\boldsymbol{f}$, The trained distribution $(\square)$ is shifted to the right of the untrained distribution $(\square)$; untrained spines predominate in smaller bins, whereas trained spines prevail in larger bins. Gray bars below represent the percentage of trained and percentage of untrained spines for each bin.

$\left(121.4 \pm 1.1 \%\right.$ trained vs $169.2 \pm 1.2 \%$ untrained; $n=5 ;{ }^{\star} p=$ 0.016) (Fig. 2c). This partial occlusion of LTP, along with larger baseline responses, suggests that skill learning induced an LTPlike process in the trained hemisphere, reducing further LTP induction.

To test whether learning a new motor skill alters synaptic structure, we analyzed dendritic spines in layer 1 of forelimb motor cortex slices. After training, slices were prepared and the baseline strength of synaptic connections measured as above. After $5 \mathrm{~d}$ of training, there was no difference in spine number $(1.83$ vs 1.97 spines $/ \mu \mathrm{m}$ median in six rats, $n=63$ dendrites untrained, 57 dendrites trained; Kruskal-Wallis, $p=0.1$ ) or spine length ( 1.56 vs $1.56 \mu \mathrm{m}$ median in six rats, $n=1476$ spines untrained, 1174 spines trained; Kruskal-Wallis, $p=0.88)$. However, spine heads were wider in the trained hemisphere (780 vs $810 \mathrm{~nm}$ median; Kruskal-Wallis, $p=0.02$ ) (Fig. $2 e$ ). Looking at the percentage of spines that fall into $0.2 \mu \mathrm{m}$ bins, the distribution of spines from the trained cortex is shifted to the right. If the percentage of untrained spines in each bin is subtracted from the percentage of trained spines in that bin, it is clear that untrained spines predominate in the smaller width bins, whereas the trained spines predominate in the larger width bins (Fig. $2 f$ ). These results are noteworthy because LTP-induced increases in spine dimensions have been reported (for review, see Harms and Dunaevsky, 2007), and at this point further LTP induction is occluded (Fig. $2 c)$.

Previously, recordings in layer $2 / 3$ showed that baseline synaptic responses remain enhanced months after training (RioultPedotti et al., 2007). We found the same to be true in layer 1; FP amplitude enhancement persisted $30 \mathrm{~d}$ without additional training $(1.06 \pm 0.06 \mathrm{mV}$ trained vs $0.71 \pm 0.05 \mathrm{mV}$ untrained for threshold factor $4 ; n=19 ;{ }^{\star} p=0.01$ ) (Fig. $3 b$, supplemental Fig. 1 , available at www.jneurosci.org as supplemental material). In contrast, LTP (after saturation) in the trained hemisphere was similar to LTP in the untrained hemisphere (149.2 $\pm 1.3 \%$ vs $146.4 \pm 1.6 \% ; n=5 ; p=0.401$ ) (Fig. $3 c$ ). This parallels findings in layer $2 / 3$, showing recovery of the synaptic modification range (Rioult-Pedotti et al., 2007).

Thirty days after training, analysis of dendritic spine morphology showed no significant difference in spine width (780 vs $740 \mathrm{~nm}$ median; Kruskal-Wallis, $p=0.19$ ) (Fig. 3e), length (1.51 vs $1.49 \mu$ m median; Kruskal-Wallis, $p=0.18$ ), or density (2.06 vs 
a

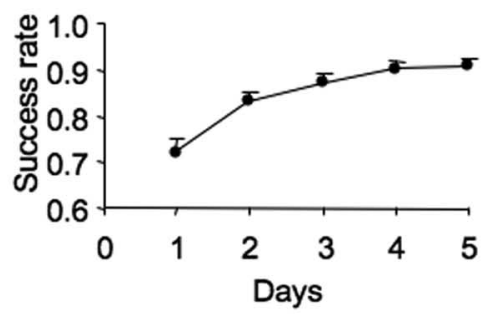

C

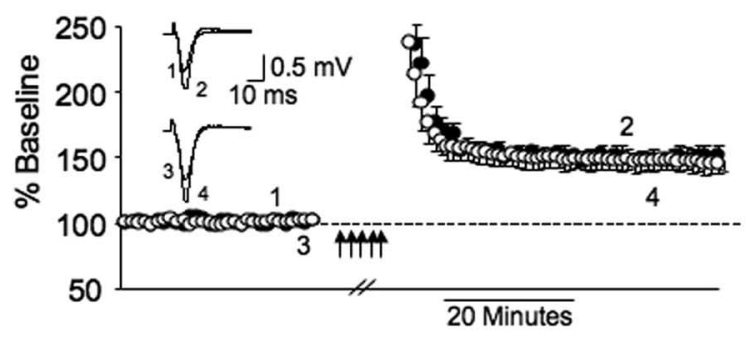

d

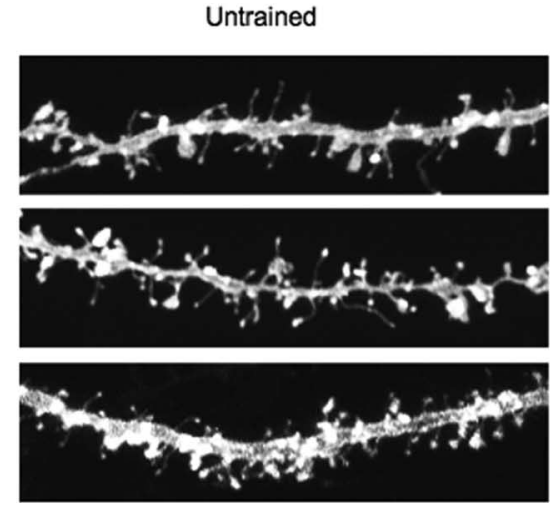

b

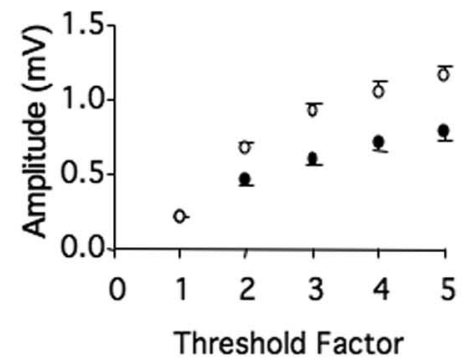

e

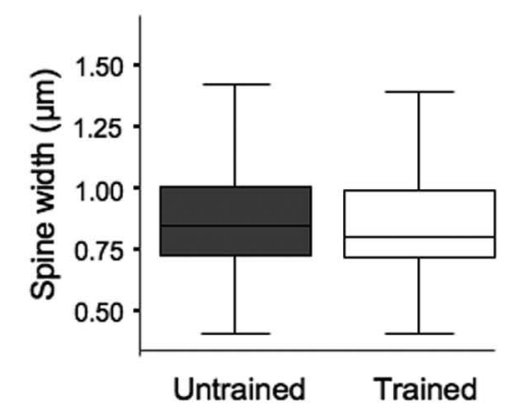

f
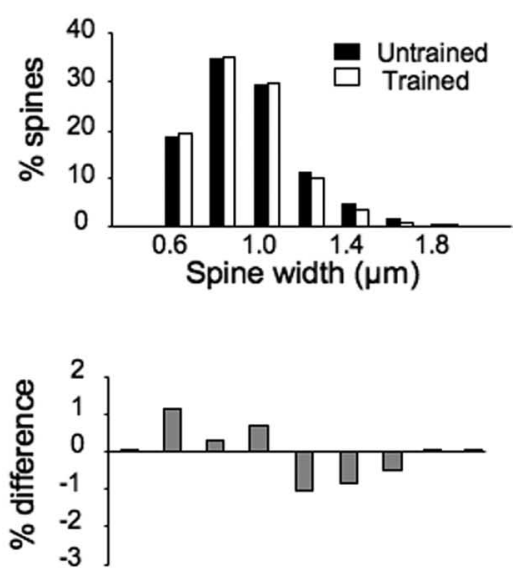

Figure 3. Response size increase remains $30 \mathrm{~d}$ after training, whereas LTP and spine width recover. $\boldsymbol{a}$, Rats learned the reach-and-grasp task over $5 \mathrm{~d}$ then returned to normal housing for $25-30$ d. $n=15 . \boldsymbol{b}$, FPs recorded simultaneously in both hemispheres indicate that the increase in FP amplitude in the trained hemisphere is maintained $30 \mathrm{~d}$ later. c, LTP was induced repeatedly (multiple arrows) until responses were saturated in both hemispheres. LTP after saturation in the trained hemisphere $(\bigcirc)$ was similar to LTP in the untrained hemisphere $(-30 \mathrm{~d}$ after motor skill training $(t$ test, $p=0.401 ; n=5)$. Inset, Individual traces before and after LTP saturation. $\boldsymbol{d}$, After recording, slices were fixed, labeled with Dil, and spines in layer 1 were imaged with confocal microscopy. $n=10$ rats. Scale bar, $10 \mu \mathrm{m} . \boldsymbol{e}$, Thirty days after training, analysis of dendritic spine morphology showed that the dendritic spine width was not different in trained and untrained hemispheres (Kruskal-Wallis, $p=0.19$ ). $f$, The trained distribution of dendritic spine width is no longer shifted. Gray bars below represent the percentage of trained and percentage of untrained spines for each bin.

2.06 spines/ $\mu \mathrm{m}$ median, Kruskal-Wallis, $p=0.85$ ) between the trained and untrained hemispheres (in 10 rats, $n=99$ dendrites and 1659 spines untrained; $n=87$ dendrites and 1432 spines trained). Looking at the percentage of spines that fall into $0.2 \mu \mathrm{m}$ bins, the distribution of spines from the trained cortex is no longer shifted (Fig. 3f). Therefore, the morphology follows the recovery of LTP in this late time point, suggesting that plasticity has been reset and the system is ready for further learning.

\section{Discussion}

We found that motor learning increases synaptic strength in the horizontal connections of layer 1 . After $5 \mathrm{~d}$ of training, LTP is partially occluded and spines are wider in layer 1 . This is the first demonstration of learning-induced changes in behavior, synaptic strength and plasticity, and structural plasticity in the same animal, and supports the idea that an LTP-like process in the primary motor cortex mediates the initial learning of a skilled task. Although the change we observe in spine size is smaller than that observed with LTP induction (Lang et al., 2004; Matsuzaki et al., 2004; Zhou et al., 2004), in those studies it was possible to follow particular potentiated synapses before and after induction. Here, we examine populations, and do not follow individual spines, or know which and how many synapses are potentiated. In addition, we use behavior to induce plasticity, not electrical or chemical induction methods. Because the learning-induced change in spine size reported here does not establish causation and does not fully explain a 50\% increase in synaptic strength, as measured by FP amplitudes, other mechanisms could contribute to the FP enhancement, such as modification of neuronal excitability, or changes in inhibition.

Learning-induced increase in spine density was reported previously in the hippocampus (Moser et al., 1994; Leuner et al., 2003) and motor cortex (Kleim et al., 2004). Learning of a skilled reaching task increased distal forelimb representation after $10 \mathrm{~d}$ and synapse formation after $7 \mathrm{~d}$ in layer 5 , suggesting that motor map reorganization and synapse formation contribute to consolidation of learning during the late stages of training (Kleim et al., 2004). We see no change in synapse number in layer 1 resulting from $5 \mathrm{~d}$ of training. Although the differences in our results may be attributable to the layer or task used in the studies, they suggest 
that if there is a change in synapse number associated with skill learning, it is a transient one.

Thirty days later, whereas both LTP and spine size return to untrained levels, baseline synaptic responses remain elevated in the trained hemisphere. What maintains the increase in baseline transmission if not potentiation of synaptic transmission, as indicated initially by LTP occlusion and increase in spine width? Our results suggest that other changes mediate the recovery of LTP with sustained baseline augmentation. We cannot discern whether the enlarged spines have been eliminated, or if a new subset of smaller spines offsets the initial spine width increase. It may be that a subset of spines remains enlarged and a second population of spines has turned over, replaced with smaller and more dynamic spines (Trachtenberg et al., 2002; Majewska and Sur, 2003; Holtmaat et al., 2006). Perhaps selective spine pruning and turnover play a larger role in this maintenance after LTP recovery. Finally, the percentage of neurons participating in the FP enhancement is unknown. If task learning initially affects connections throughout the cortex, but is then maintained by more sparse connections, these changes may be overlooked in population studies, especially if learning of other tasks continues to change spines in both hemispheres. To address these questions, monitoring of specific spines over time is necessary.

\section{References}

Bourne J, Harris KM (2007) Do thin spines learn to be mushroom spines that remember? Curr Opin Neurobiol 17:381-386.

Cauller L (1995) Layer I of primary sensory neocortex: where top-down converges upon bottom-up. Behav Brain Res 71:163-170.

Douglas RJ, Martin KA (2004) Neuronal circuits of the neocortex. Annu Rev Neurosci 27:419-451.

Evans HM, Long JA (1922) Characteristic effects upon growth, oestrus and ovulation induced by the intraperitoneal administration of fresh anterior hypophyseal substance. Proc Natl Acad Sci USA 8:38-39.

Gan WB, Grutzendler J, Wong WT, Wong RO, Lichtman JW (2000) Multicolor "DiOlistic" labeling of the nervous system using lipophilic dye combinations. Neuron 27:219-225.

Harms KJ, Dunaevsky A (2007) Dendritic spine plasticity: looking beyond development. Brain Res 1184:65-71.
Hodgson RA, Ji Z, Standish S, Boyd-Hodgson TE, Henderson AK, Racine RJ (2005) Training-induced and electrically induced potentiation in the neocortex. Neurobiol Learn Mem 83:22-32.

Holtmaat A, Wilbrecht L, Knott GW, Welker E, Svoboda K (2006) Experience-dependent and cell-type-specific spine growth in the neocortex. Nature 441:979-983.

Kleim JA, Hogg TM, VandenBerg PM, Cooper NR, Bruneau R, Remple M (2004) Cortical synaptogenesis and motor map reorganization occur during late, but not early, phase of motor skill learning. J Neurosci 24:628-633.

Lang C, Barco A, Zablow L, Kandel ER, Siegelbaum SA, Zakharenko SS (2004) Transient expansion of synaptically connected dendritic spines upon induction of hippocampal long-term potentiation. Proc Natl Acad Sci USA 101:16665-16670.

Leuner B, Falduto J, Shors TJ (2003) Associative memory formation increases the observation of dendritic spines in the hippocampus. J Neurosci 23:659-665.

Majewska A, Sur M (2003) Motility of dendritic spines in visual cortex in vivo: changes during the critical period and effects of visual deprivation. Proc Natl Acad Sci USA 100:16024-16029.

Matsuzaki M, Honkura N, Ellis-Davies GC, Kasai H (2004) Structural basis of long-term potentiation in single dendritic spines. Nature 429:761-766.

Moser MB, Trommald M, Andersen P (1994) An increase in dendritic spine density on hippocampal CA1 pyramidal cells following spatial learning in adult rats suggests the formation of new synapses. Proc Natl Acad Sci USA 91:12673-12675.

Rioult-Pedotti MS, Friedman D, Hess G, Donoghue JP (1998) Strengthening of horizontal cortical connections following skill learning. Nat Neurosci 1:230-234.

Rioult-Pedotti MS, Friedman D, Donoghue JP (2000) Learning-induced LTP in neocortex. Science 290:533-536.

Rioult-Pedotti MS, Donoghue JP, Dunaevsky A (2007) Plasticity of the synaptic modification range. J Neurophysiol 98:3688-3695.

Sanes JN, Donoghue JP (2000) Plasticity and primary motor cortex. Annu Rev Neurosci 23:393-415.

Trachtenberg JT, Chen BE, Knott GW, Feng G, Sanes JR, Welker E, Svoboda $\mathrm{K}$ (2002) Long-term in vivo imaging of experience-dependent synaptic plasticity in adult cortex. Nature 420:788-794.

Zhou Q, Homma KJ, Poo MM (2004) Shrinkage of dendritic spines associated with long-term depression of hippocampal synapses. Neuron 44 $749-757$. 